\title{
33. METEOR SHOWER MASS DISTRIBUTION FROM RADAR ECHO COUNTS
}

\author{
W. G. ELFORD \\ (Dept. of Physics, University of Adelaide, Australia)
}

\begin{abstract}
Theoretical hourly rates of radar echoes from meteor showers observed with narrow- and wideaperture systems are calculated in terms of equipment parameters and the distribution of the number of meteors as a function of mass. From a comparison of the echo rates in selected range intervals, it is shown that it is possible to determine the value of the exponent in a simple inverse power flux law.

The effect on the echo rate of a change in the value of the exponent in the flux law with magnitude is described and the probability of detecting showers at different stations is discussed.

It is suggested that simple radar echo counts in several range intervals can be used to determine the mass distribution of meteor showers over a range of two or three magnitudes above the limiting magnitude of the system. The analysis is applied to some observations made at Christchurch and Ottawa.
\end{abstract}

\section{Introduction}

The rate of radar echoes from a meteor shower is dependent on the number distribution of trails as a function of maximum electron line density. If it is assumed that the maximum line density is proportional to the mass of the meteoroid then it is possible to obtain information concerning the mass distribution from a study of radar echo rates. Previous workers (Browne et al., 1956; Weiss, 1961; Kaiser, 1961 ; McIntosh, 1966) have determined the mass distribution of six major meteor showers from radio observations of meteor echo rates, echo durations and trail-height distributions. In each case the mass distribution has been determined in terms of an assumed inverse power law distribution over a limited magnitude range. The results are not all consistent but in general they show that for the majority of showers the exponent in the power law varies rapidly with magnitude between radio magnitudes 0 and +8 .

In this paper the manner in which the echo rate for a given radar system and shower radiant varies with the choice of the mass distribution is examined theoretically and the results compared with observed rates. It is shown that echo rates for both narrow- and wide-aperture systems can be used to infer the mass distribution of an observed shower. The essence of the method for both types of systems is the comparison of echo rates in selected range intervals. In addition, for the wide-aperture 
system, the shape of the total echo rate vs. time curve can give a measure of the distribution law, but this is less precise than the method of selected range intervals.

\section{Theoretical Echo Rates}

Theoretical echo rates from meteor showers have been calculated for three radar systems and several forms of the mass distribution. The three systems that have been investigated are:

(1) the wide-aperture patrol radar on $32.7 \mathrm{mc} / \mathrm{s}$ at Ottawa, $45^{\circ} \mathrm{N}$ (Neale, 1966);

(2) the wide-aperture patrol radar on $69 \mathrm{mc} / \mathrm{s}$ at Christchurch, $43 \cdot 5^{\circ} \mathrm{S}$ (Ellyett and Keay, 1963);

(3) a narrow-beam low-angle system on $69 \mathrm{mc} / \mathrm{s}$ at Christchurch (Ellyett et al., 1961).

The response of each system to a radiant in a given position in the sky has been calculated according to a method developed by Elford (1964), but modified to include overdense trails, any form of the distribution of ionization along a trail, and any range interval used in recording the data. It is assumed that reflection is specular and that the zenithal line density $q_{z}$ is related to the actual line density by a simple $\cos z$ factor, where $z$ is the zenith distance of the radiant. The distribution of zenithal electron line density along a meteor trail is assumed to be gaussian with a maximum at $95 \mathrm{~km}$ and a standard deviation of 6 or $12 \mathrm{~km}$. Two types of mass distributions have been investigated; (A) a simple inverse-power law with a constant exponent over all magnitudes, and (B) an inverse-power law with an exponent that can vary with magnitude.

\section{A. SIMPLE INVERSE-POWER LAW DISTRIBUTION, CONSTANT EXPONENT}

In the first analysis, echo rates were calculated for selected range intervals on the assumption that the mass-distribution law was a simple inverse power, i.e. the number of trails, $\mathrm{d} N\left(q_{\mathrm{z}}\right)$ with maximum zenithal line densities between $q_{\mathrm{z}}$ and $q_{\mathrm{z}}+\mathrm{d} q_{\mathrm{z}}$ is assumed to be given by:

$$
\mathrm{d} N\left(q_{\mathrm{z}}\right) \propto q_{\mathrm{z}}^{-s} \mathrm{~d} q_{\mathrm{z}} .
$$

By integration, the number of trails with maximum zenithal line density equal or greater than $q_{\mathrm{z}}$ is given by:

$$
N\left(q_{\mathrm{z}}\right) \propto q_{\mathrm{z}}^{1-s}
$$

It is convenient to write this cumulative law as:

$$
N=K q_{\mathrm{z}}^{c}, \text { where } c=1-s .
$$

Rates were calculated for three values of $c:-0 \cdot 6,-1 \cdot 0,-1 \cdot 4$. The results for the narrow-beam system and a radiant with declination $-20^{\circ}$ are shown in Figure 1 . Five range intervals, each $100 \mathrm{~km}$ wide and commencing at a range of $350 \mathrm{~km}$, 
were selected. The effect of small minor lobes in the antenna polar diagram were not included in the calculation of the rates, as a simple analysis showed that echoes due to these lobes would be limited to ranges less than $250 \mathrm{~km}$. The echo rate vs. time curves have been normalized to the peak rate in the $350-450 \mathrm{~km}$ range interval.

$350 \cdot 450$ KMS

$450 \cdot 550$ KMS

$550-c 50 \quad r .15$

$650 \cdot 750$ KMS

$750-850$ KMS
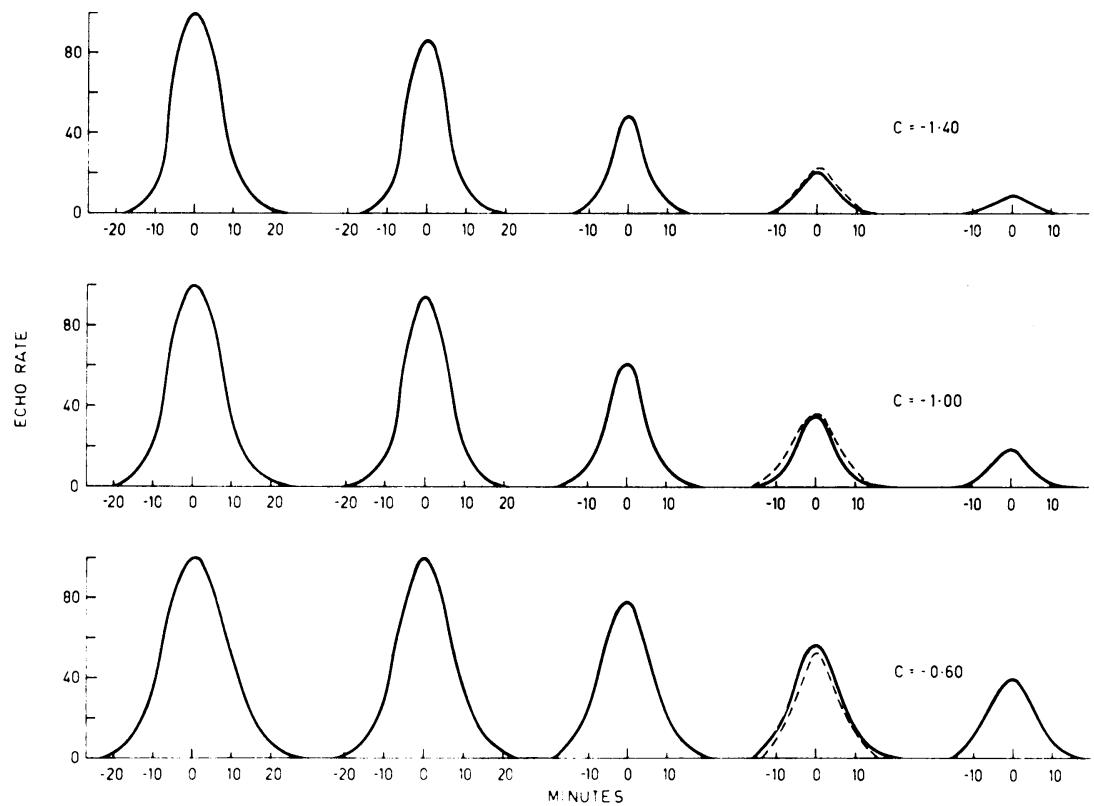

IIME IRELATIVE TO PEAK RAIEI

FIG. 1. Theoretical echo rates in five selected range intervals and for three values of $c$. Radiant declination $-20^{\circ}$; Christchurch narrow-beam system. For dashed curve, see text.

The rate curves were calculated using a gaussian distribution of ionization along the trails with a standard deviation of $6 \mathrm{~km}$. The effect of doubling the standard deviation to $12 \mathrm{~km}$ is shown by the dashed curves for the $650-750 \mathrm{~km}$ range interval. It is evident that the form of the ionization distribution has only a marginal effect on the echo rate.

The most significant feature of the curves in Figure 1 is the manner in which the peak rate decreases with increasing range for different values of the exponent $c$ in the distribution law. The variation is most marked at long ranges. In the range interval $750-850 \mathrm{~km}$, the peak rate for $c=-0.6$ is $40 \%$ of the rate in the $350-450 \mathrm{~km}$ interval as compared with a peak rate of $10 \%$ for $c=-1 \cdot 4$. The variation of the peak rate with the value of $c$ is readily compared by drawing a locus through the peak-rate values, as shown in Figure 2. Observations carried out with the narrow-beam system at Christchurch (Ellyett et al., 1961) show that for the $\delta$-Aquarid shower (declination 
$\sim-18^{\circ}$ ) the relative echo rates in the $350-450$ and $450-550 \mathrm{~km}$ range intervals are almost equal, as predicted by the theoretical calculations. Unfortunately, the echo counts have not been given for the longer ranges and thus no conclusion can be drawn concerning the form of the mass distribution from the published observations.

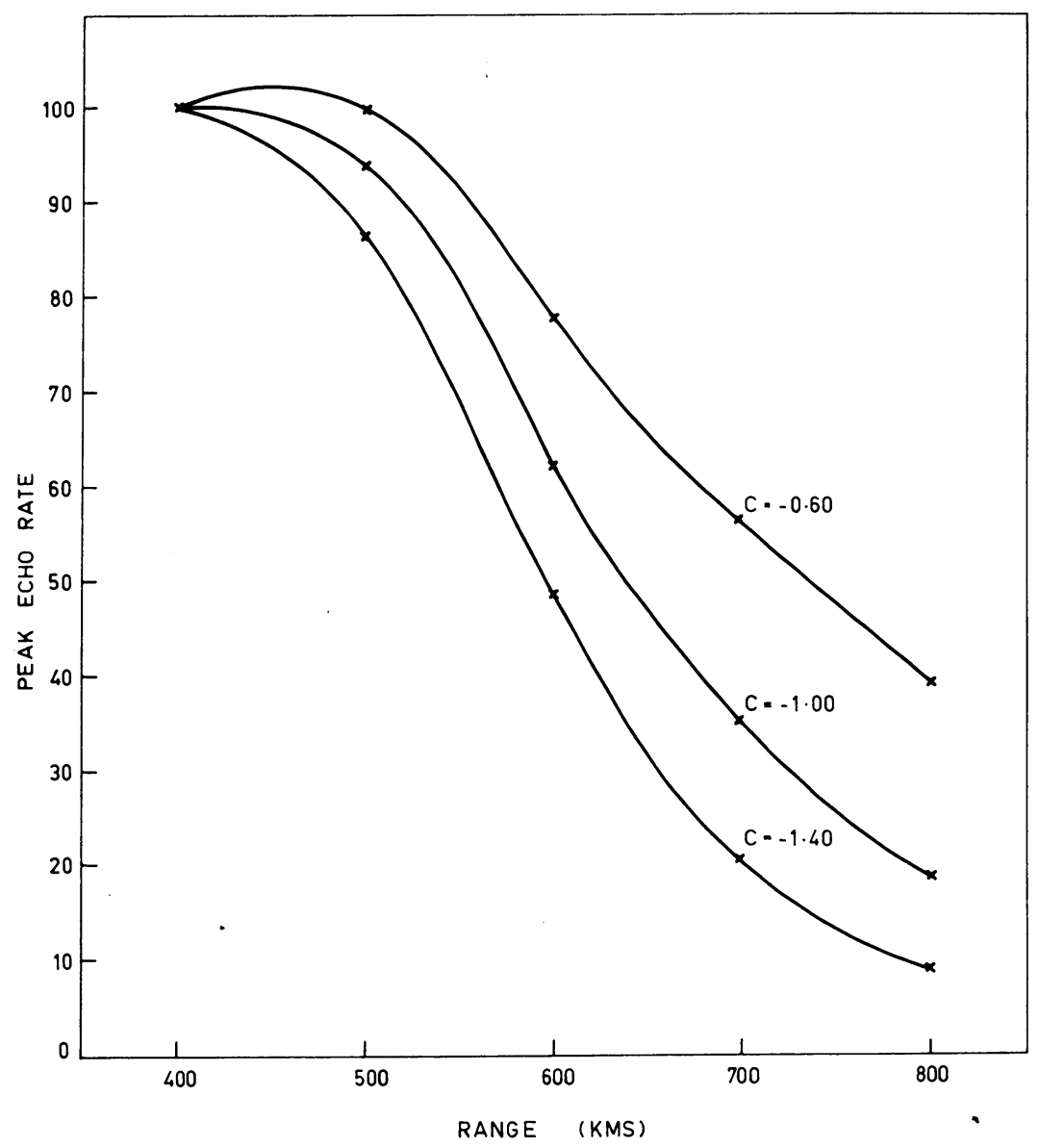

FIG. 2. Locus of peak rates shown in Figure 1.

Theoretical echo rates have also been calculated for the wide-aperture system at Christchurch. Echo rate vs. time curves for a radiant at declination $-60^{\circ}$, and echoes in the range intervals 100-200, 200-300 and 300-400 km are shown in Figure 3. For this relatively high declination radiant echoes are detected for the whole day, although for ranges $>200 \mathrm{~km}$ the width of the rate curves at half the peak rate is about 6 hours. Again it can be seen that a comparison of the rates in the 100-200 and $300-400 \mathrm{~km}$ range intervals will give a measure of the exponent in the mass-distri- 
bution law. An examination of the rate-time curves for radiants at all declinations visible from Christchurch shows that the change in the rate with range is a sensitive indicator of the mass distribution.

One disadvantage of the wide-aperture system for investigating the mass distribution
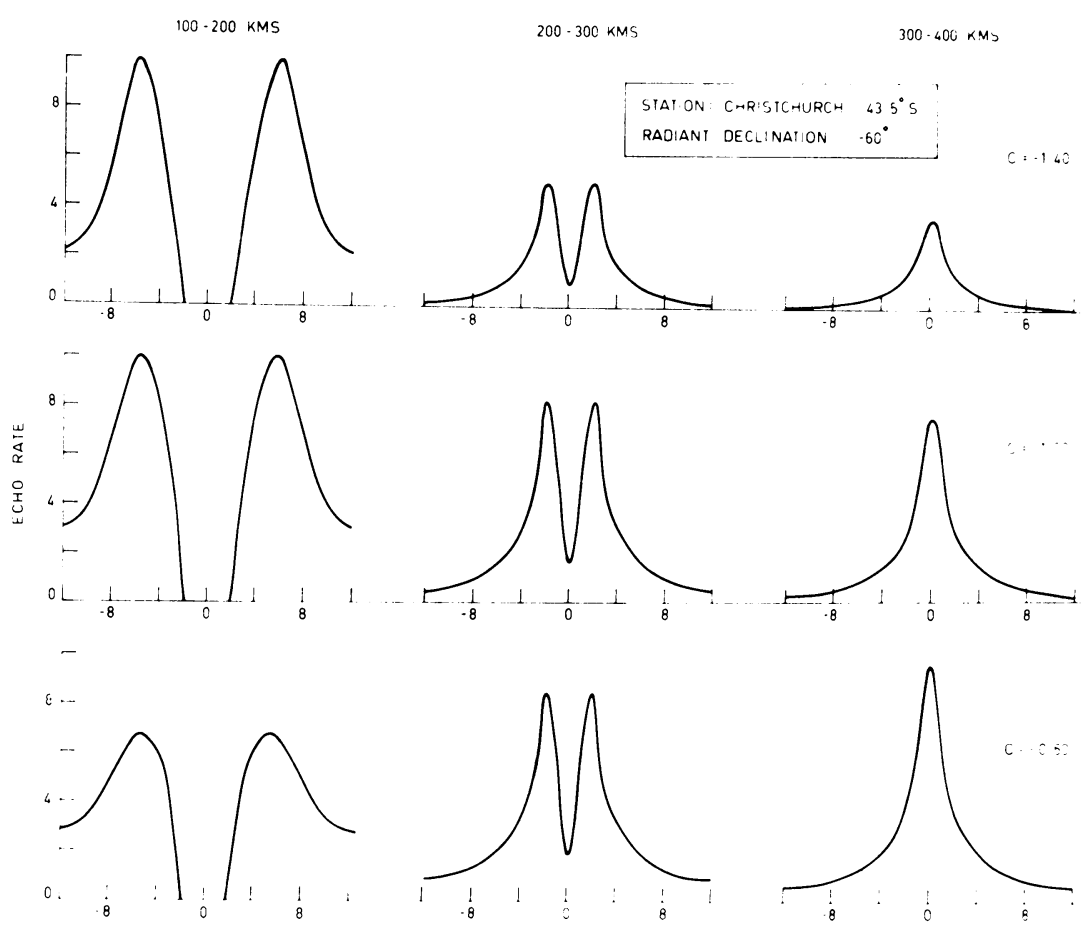

FIG. 3. Theoretical echo rates in three selected range intervals and for three values of c. Christchurch wide-aperture system.

of showers is that the rate data must be collected over about 12 hours, and thus the analysis discussed above can only apply to showers whose mass distribution is approximately constant for this period of time. On the other hand, the narrow-beam system discussed earlier detects echoes from the majority of meteor showers in a time interval of less than $1 \frac{1}{2}$ hours.

\section{B. INVERSE-POWER LAW DISTRIBUTION, VARIABLE EXPONENT}

As indicated earlier, previous investigations of the mass distribution of a number of major showers indicate, that the exponent in the power law distribution varies with magnitude. To examine the effect of a varying exponent on the rate-time curves, theoretical echo rates were calculated for the three radar systems being considered. 
The distribution functions were generated as follows: For any limiting value of the zenithal line density $q_{\mathrm{z}}$ it was assumed that the cumulative distribution law could be written as:

$$
\log _{10} N=\sum_{i=0}^{n} a_{i} M_{R}^{i}
$$

where $M_{R}=40-2 \cdot 5 \log _{10} q_{z}$.

The only information available on the behaviour of such a distribution function is the published values of the exponent $c$ for an assumed simple inverse-power law over limited ranges of $M_{R}$.

Now

$$
c=-2 \cdot 5 \frac{\mathrm{d}\left(\log _{10} N\right)}{\mathrm{d} M_{R}}=-2 \cdot 5 \sum_{i=1}^{n} i a_{i} M_{R}^{i-1} .
$$

The value of $n$ was chosen as 5 , and the coefficients $a_{i}$ which gave an expression that best matched the experimental curve of $c$ vs. $M_{R}$ were determined by the method of least squares.

In order to compare the effects of these new variable distribution functions on the echo rate it is convenient to consider the rate for a point source radiant as a function of the elevation and azimuth of the radiant relative to the station. This description of the echo rate as a function of radiant position is generally termed the 'response function' of the system. For the wide-aperture systems at Ottawa and Christchurch the antenna polar diagrams are independent of azimuth, and hence the response functions for these systems depend only on radiant elevation. The response functions for these two systems have been calculated for a number of values of $c$ and in particular for the Arietid and $\delta$-Aquarid showers, both of which show a change in the value of the exponent in the mass distribution with magnitude. The results of the latter calculations are shown in Figure 4 together with the experimental curves of $c$ vs. $M_{R}$ for both showers. The marked difference in the positions of the peak of the two response functions in the case of the Christchurch system is due to a minor lobe at a low angle of elevation.

\section{Comparison of Theoretical and Observed Rates}

The theoretical radar echo rate as a function of time for the Arietid and $\delta$-Aquarid showers can readily be deduced from the response functions plotted in Figure 4, and the results are shown in Figures 5 and 6. The graphs indicate that the Ottawa system should detect about five times as many Arietid meteors and half as many $\delta$-Aquarid meteors as the Christchurch system. At the present time there are no published shower rates that can be compared with these predictions. However, from the echo rate data of Ellyett and Keay (1963) hourly echo rates for the $\delta$-Aquarid shower averaged over the three days July 25-27, 1960 were determined, and the normalized values are shown at hourly intervals in Figure 6. It is evident that the theo- 

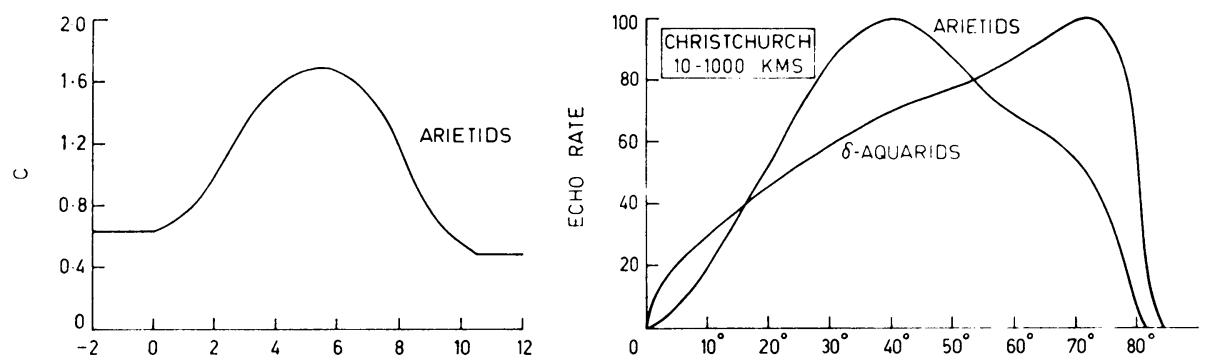

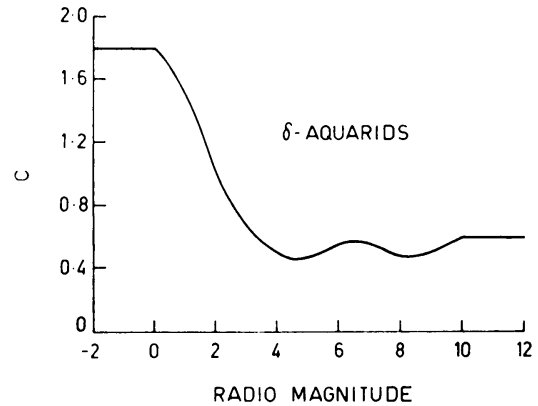

(a)

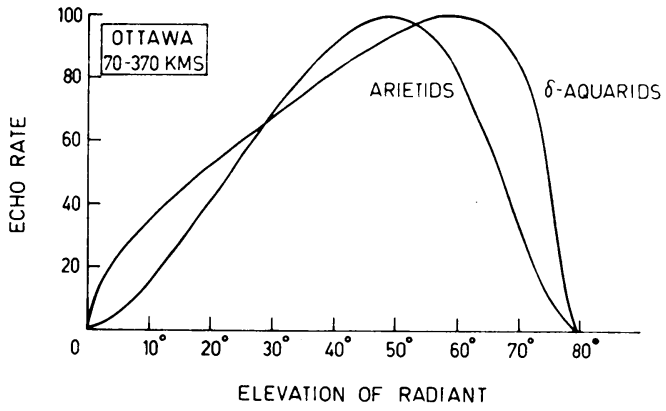

(b)

FIG. 4. (a) Variation of mass-distribution exponent with radio magnitude for Arietid and $\delta$-Aquarid showers. - (b) Normalized response functions for the wide-aperture systems at Christchurch and Ottawa calculated for the mass distribution of $(a)$.

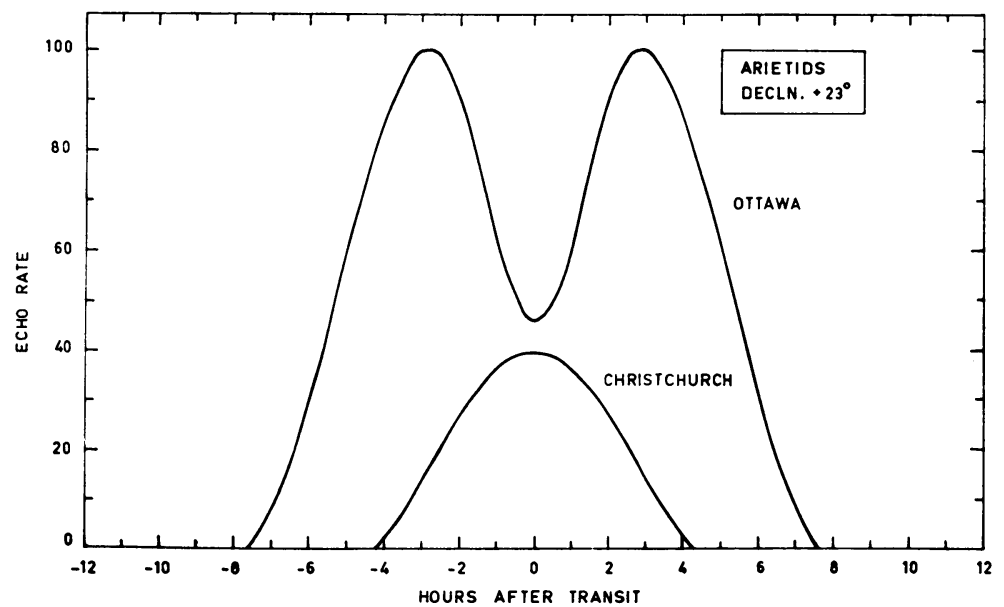

Fig. 5. Theoretical echo rates of Arietid meteors observed at Ottawa and Christchurch. Normalized to peak rate at Ottawa.

retical echo-rate curve is too wide. The fit could be improved if the value of $c$ for $M_{R}>5$ was chosen as $-0 \cdot 4$. Alternatively it is possible that the variation of the observed 
echo rate with time would be different if the long-duration echoes were removed. It has been shown by McIntosh (1966) that in the case of the Geminid shower the rate-time curve is significantly affected by long-duration non-specular echoes. Thus any conclusions to be drawn from the Christchurch echo rates concerning the $\delta$ Aquarid mass distributions must be considered as tentative until the hourly rates of the short-duration $\delta$-Aquarid echoes are known.

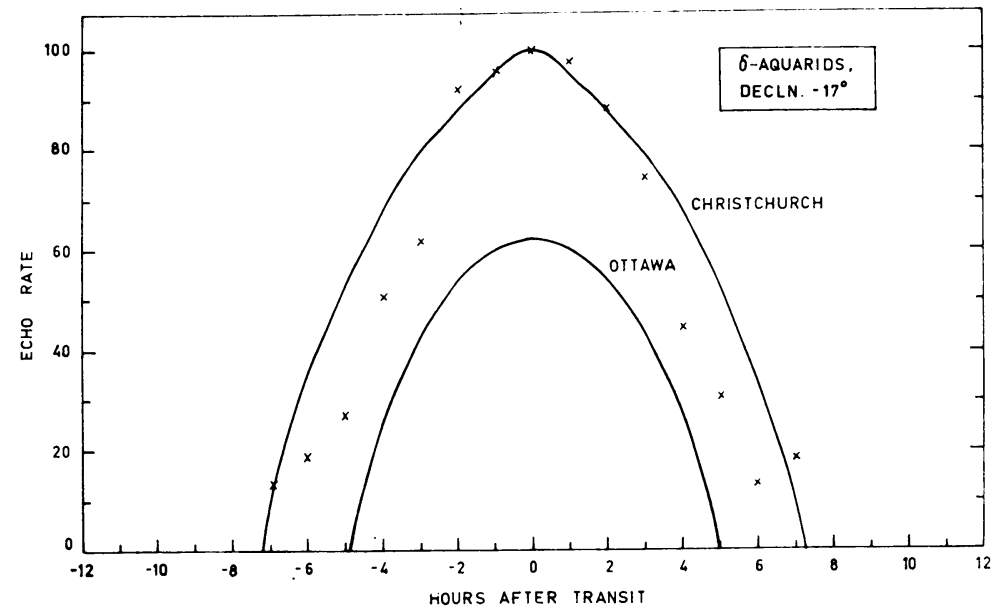

FIG. 6. Theoretical echo rates of $\delta$-Aquarid meteors observed at Christchurch and Ottawa. Normalized to peak rate at Christchurch. Crosses show normalized hourly rates observed at Christchurch, July 25-27, 1960.

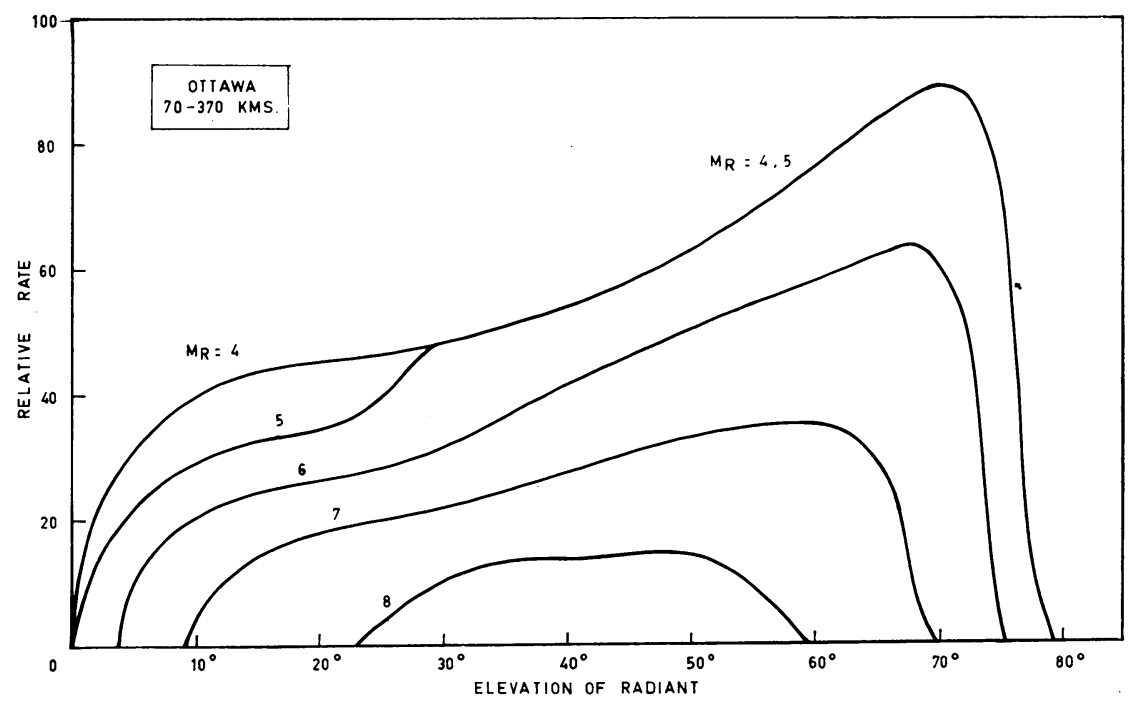

FIG. 7. Response functions for the Ottawa system for a constant flux of meteors at radio magnitudes $+4,+5,-6,+7$ and +8 . 
McIntosh (1966) has examined the radio echo rate from the Geminid shower observed at Ottawa on December 13,1964 and has shown that the normalized rate of short-duration echoes as a function of radiant elevation cannot be matched with a theoretical response function with a constant value of the exponent $c$ in the mass distribution. In the present investigation an attempt was made to synthesize an appropriate response function for the Geminid shower by combining the weighted responses of the Ottawa system to meteors of radio magnitude $+4,+5,+6,+7$ and +8 . The response of the Ottawa system to a constant flux over a range of $0 \cdot 1$ magnitudes centered on each of these five values of radio magnitude is shown in Figure 7. These graphs show that (1) echoes at times of high and low elevations of the radiant are contributed by the brighter meteors, (2) the peak rate occurs at lower elevations of the radiant as the mass decreases, and (3) over a limited range of radiant elevations the Ottawa system can detect meteors down to radio magnitude +8 . However, it was found impossible to synthesize a response function from these curves that would match the observed echo rate as a function of radiant elevation, and it is concluded that the inconsistency is due to a change in the mass distribution of the Geminids during the hours of observations.

\section{Conclusions}

Simple counts of short-duration radar echoes in selected range intervals can be used to determine the mass distribution of a meteor shower over a range of two or three magnitudes above the limiting magnitude of the system. This method is particularly applicable to narrow-aperture systems which detect echoes from a shower within a relatively short period of time.

\section{Acknowledgements}

The author is indebted to B. J. Stone for assistance with the computations and for valuable comments.

This work was supported in part by the National Aeronautics and Space Administration through grant NGR-52-042-003.

\section{References}

Browne, I., Bullough, K., Evans, S., Kaiser, T.R. (1956) Proc. phys. Soc., 69B, 84. Elford, W.G. (1964) Harvard-Smithsonian Radio Meteor Project Res. Rep., No. 8. Ellyett, C., Keay, C.S.L., Roth, K.W., Bennett, R.G.T. (1961) Mon. Not. R. astr. Soc., 123, 37. Ellyett, C., Keay, C.S.L. (1963) Mon. Not. R. astr. Soc., 125, 325.

Kaiser, T.R. (1961) Ann. Géophys., 17, 50.

McIntosh, B. A. (1966) Can. J. Phys., 44, 2729.

Neale, M.J. (1966) Can.J. Phys., 44, 1021.

Weiss, A. A. (1961) Rust. J. Phys., 14, 102. 


\section{DISCUSSION}

Glöde: If rates of long-enduring echoes have to be reduced due to the mentioned influence of echoes non-specular at their beginning, the total mass influx of shower meteors becomes still smaller compared with the sporadic background, so that no extraordinary geophysical effects connected with the showers should be expected.

Elford: In the report of the symposium on Meteor Orbits and Dust held in Boston in 1965 you will find a table of influx rates of shower and sporadic meteors that I prepared. I believe that it shows that sporadic meteors dominate over all showers for meteors fainter than magnitude +5 . 